\title{
Kinematics Analysis and Simulation of a Novel 3T Parallel Mechanism
}

\author{
Yongdong Huang $\mathbb{D}^{1},{ }^{1}$ Quanguo Lu $\mathbb{D}^{1},{ }^{1}$ Hongzhou Wang $\mathbb{D}^{2,3}$ Jinfeng Liu $\mathbb{D}^{1},{ }^{1}$ Zhihao Li $\mathbb{D}^{1}{ }^{1}$ \\ Xiaohui Zou $\mathbb{D}^{2},{ }^{2}$ and Xiaohuang Zhan $\mathbb{D}^{2}$ \\ ${ }^{1}$ Jiangxi Province Key Laboratory of Precision Drive and Control, Nanchang Institute of Technology, Nanchang, China \\ ${ }^{2}$ Jiangxi Institute of Mechanical Science, Nanchang, China \\ ${ }^{3}$ School of Mechatronic Engineering and Automation, Shanghai University, Shanghai, China
}

Correspondence should be addressed to Quanguo Lu; luqg2010@126.com

Received 8 November 2021; Revised 9 December 2021; Accepted 3 January 2022; Published 7 March 2022

Academic Editor: Jaime Gallardo Alvarado

Copyright ( $) 2022$ Yongdong Huang et al. This is an open access article distributed under the Creative Commons Attribution License, which permits unrestricted use, distribution, and reproduction in any medium, provided the original work is properly cited.

\begin{abstract}
Three translational (3T) degree-of-freedom (DOF) parallel mechanism is widely used in the industrial field because of its compact structure and excellent dynamic performance. Combined with the 3T parallel mechanism studied at this stage, a novel 3T parallel mechanism is proposed. Firstly, the DOF of the mechanism is analyzed by using the screw theory. Secondly, the position, velocity, and singularity of the mechanism are analyzed, and the workspace of the mechanism is analyzed through the positive position solution. Then, the trajectory planning in joint space and coordinate space is completed, and a common mathematical model of grasping trajectory is established. Thirdly, the kinematics simulation analysis of the mechanism is carried out. Finally, the prototype platform was built and the grasping experiment was carried out, which verified the rationality of the mechanism design and the correctness of the theoretical analysis.
\end{abstract}

\section{Introduction}

Compared with the traditional series mechanism, the parallel mechanism has the advantages of large rigidity, compact structure, fast dynamic response, and high precision $[1,2]$. In recent years, it has become a research hot spot in industry and academia. The parallel mechanism with three translational degrees of freedom has attracted much attention because of its simple structure and can meet the requirements of industrial robots to complete various pickand-place operations in space. In 1985, the three translational delta parallel mechanism proposed by Dr. Clavel [3] has been widely used in industry [4-7]. After that, Hervé [8] proposed a 3T star mechanism. In 1996, Tsai $[9,10]$ used Hooke hinges instead of spherical hinges to simplify the delta mechanism and proposed a $3 \mathrm{~T}$ Tsai mechanism. In 2006, Liu [11-13] replaced the rotation pairs and input components of the delta parallel mechanism and Tsai mechanism with moving amplitude and slider, respectively, to obtain two linearly driven $3 \mathrm{~T}$ parallel mechanisms. In 2020, Zou [14, 15] listed a series of three DOF parallel mechanisms with parallelogram branches and mentioned that when more planar joints (moving and rotating joints) are used, higher performance can be obtained.

All of the above mechanism subchains contain parallelograms with fixed side lengths, and the parallelograms are used to limit the DOFs of rotation or enhance the mechanism performance. In recent years, some scholars have proposed and studied the parallelogram closed-loop subchain with variable rod length and thus designed a variety of new parallel mechanisms. Gogu [16] mentioned a variety of parallelogram subchains with variable rod lengths in his work and pointed out that such chains can increase the working space or improve the accuracy of the mechanism under different circumstances. In 2018, Yang $[17,18]$ proposed a new type of retractable parallelogram subchain, analyzed 3T, 3T1R, and 3R mechanisms compounded by different combinations of this type of subchains, verified 
their feasibility, and piloted a planar $2 \mathrm{~T}$ mechanism for automatic docking devices. In 2019, Yang [19] proposed a scalable parallelogram branched chain composed of collapsible kinematic chains, and based on the collapsibility of the branched chain, the authors discussed the potential application of the mechanism on UAVs, but its mechanical analysis and singularity analysis were complicated by the fact that the branched chain consisted of kinematic chains. In the same year, Wang [20] proposed a 3T parallel mechanism with a parallelogram synchronous telescopic branch chain, using a motor and a synchronous belt to make a variablelength parallelogram as the drive. However, since the drive is mounted on the branch chain, the mechanism has high rotational inertia and poor dynamics. In response to the problems arising from the above variable rod length parallelogram closed-loop subchain, this paper designs a novel 3T parallel mechanism in combination with the delta parallel mechanism [21, 22].

\section{Introduction to Parallel Mechanism}

In this paper, the $3 \mathrm{~T}$ parallel mechanism consists of a static platform, a moving platform, and three composite branches of the same structure containing parallelogram closed-loop subchains with variable rod lengths. The three composite branches are distributed on the static platform at an angle of $120^{\circ}$. The three rotation pairs of the branch chain connected to the static platform are the active pairs of the parallel mechanism, and the motor drives the compound branch chain through the active pair to realize the three translational movement of the moving platform. The mechanism is mainly composed of lightweight rods. The composite branches are symmetrically distributed and isotropic. The driving motors are fixed on the static platform, which makes the machine have good motion performance. The structure diagram of the parallel mechanism is shown in Figure 1.

As shown in Figure 2, each composite branch chain is composed of an active arm and a variable-length parallelogram closed-loop subchain connected by a rotating pair, and the variable-length parallelogram closed-loop subchain is formed by a connecting piece and a sliding rod connected by a moving pair. The active arm and the variable-length parallelogram closed-loop subchain are always maintained at $90^{\circ}$, and the parallelogram closed-loop subchain is connected with the moving platform by a spherical pair. ADEF is a parallelogram, and $\mathrm{ABCD}$ is a parallelogram with variable rod length. The lengths of rods $\mathrm{AB}$ and rod $\mathrm{CD}$ vary. $M_{\mathrm{i}}$ and $N_{\mathrm{i}}$ are the midpoints of $\mathrm{AD}$ and $\mathrm{BC}$, respectively. From the geometric relationship, we can see that $\mathrm{AB}=\mathrm{M}_{\mathrm{i}} \mathrm{N}_{\mathrm{i}}=\mathrm{CD}$. The composite branch chain is connected to the static platform through the rotating pair $L_{\mathrm{i}}$ and connected to the moving platform through two spherical hinges $B$ and $C . L_{i} M_{i}$ is perpendicular to $A D$.

\section{Degree of Freedom Analysis}

As shown in Figure 3, the parallelogram closed-loop subchain of variable rod length contains two RPS branches (R represents rotation pair, whose axis is parallel to axis $X$, and

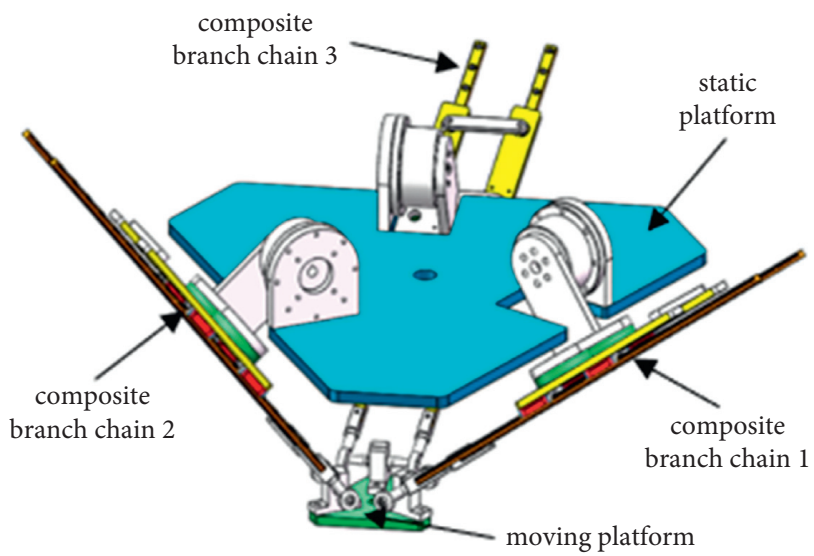

FIgURE 1: The structure diagram of parallel mechanism.

passes through point $\mathrm{A}, \mathrm{P}$ represents moving pair, whose direction is $A B, S$ represents spherical hinge, whose center is located at point $B$ ), and a parallel mechanism composed of a parallelogram closed-loop subchain ADEF where the parallelogram closed-loop subchain is to provide constraints such that $\mathrm{AB}$ and $\mathrm{CD}$ are parallel. $\mathrm{AB}=l_{1}, \mathrm{CD}=l_{2}$, $\mathrm{AE}=\mathrm{DF}=l_{3}$, and $\mathrm{BC}=\mathrm{AD}=\mathrm{EF}=l_{4}$. Given initial conditions $l_{1}=l_{2}, \beta_{i}=\pi / 2$, and the mechanism is in a singular position.

Because the rods $A B$ and $C D$ are telescopic, when the angle of the closed-loop branch reaches a singularity of the mechanism, the motion bifurcation may occur when passing through the singularity, resulting in two configurations.

As shown in Figure 4, when in configuration 1, the mechanism is a parallelogram mechanism, and the two groups of opposite edges are parallel and equal, so the rod $\mathrm{AB}$ and rod $\mathrm{CD}$ are synchronous telescopic parts.

To study the degree of freedom of this mechanism, as shown in Figure 5, when it is a fixed value, the 4-rod mechanism is regarded as a parallel mechanism with two branches, and the relative degree of freedom between its upper and lower links is studied. This closed loop can be regarded as a parallel mechanism consisting of a moving platform, a static platform, and two RS branches, with member BC as the "moving platform".

Component $\mathrm{AD}$ is the relative "static platform," $\mathrm{AB}$ is the first branch, and $\mathrm{CD}$ is the second branch. Point $\mathrm{A}$ is located on the axis of the rotation pair of the first branch. Take point $\mathrm{A}$ as the origin of the coordinate system. The axis $X$ is perpendicular to the parallelogram plane, the axis $Y$ is along the direction of $\mathrm{AD}$, and the axis $Z$ follows the righthand rule.

Branch 1 includes a spherical hinge and a rotation pair, and a spherical hinge is equivalent to three degrees of freedom of rotation perpendicular to each other, so its motion spiral system is given by

$$
\begin{aligned}
& \$_{\mathrm{A}}=\left[\begin{array}{llllll}
1 & 0 & 0 & 0 & 0 & 0
\end{array}\right]^{T}, \\
& \$_{B}:\left\{\begin{array}{l}
\$_{1}=\left[\begin{array}{llllll}
1 & 0 & 0 & 0 & f & -e
\end{array}\right]^{T}, \\
\$_{2}=\left[\begin{array}{lllll}
0 & 1 & 0 & -f & 0
\end{array}\right]^{T}, \\
\$_{3}=\left[\begin{array}{llllll}
0 & 0 & 1 & e & 0 & 0
\end{array}\right]^{T} .
\end{array}\right.
\end{aligned}
$$




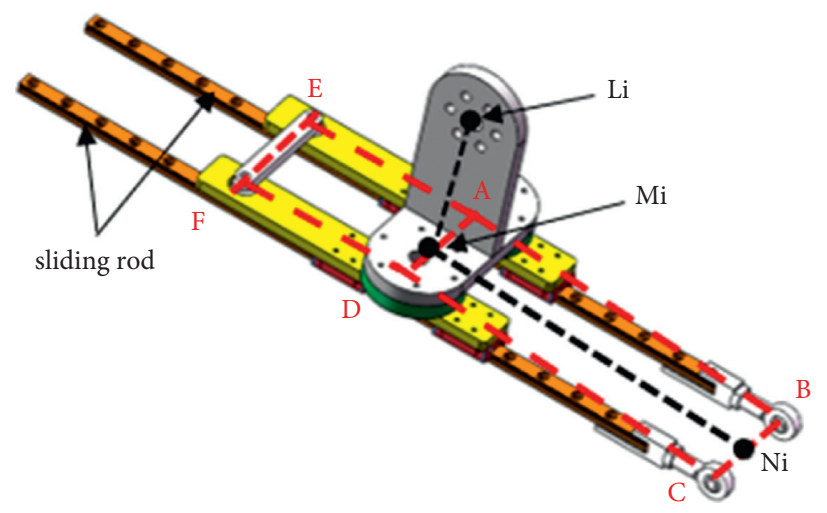

(a)

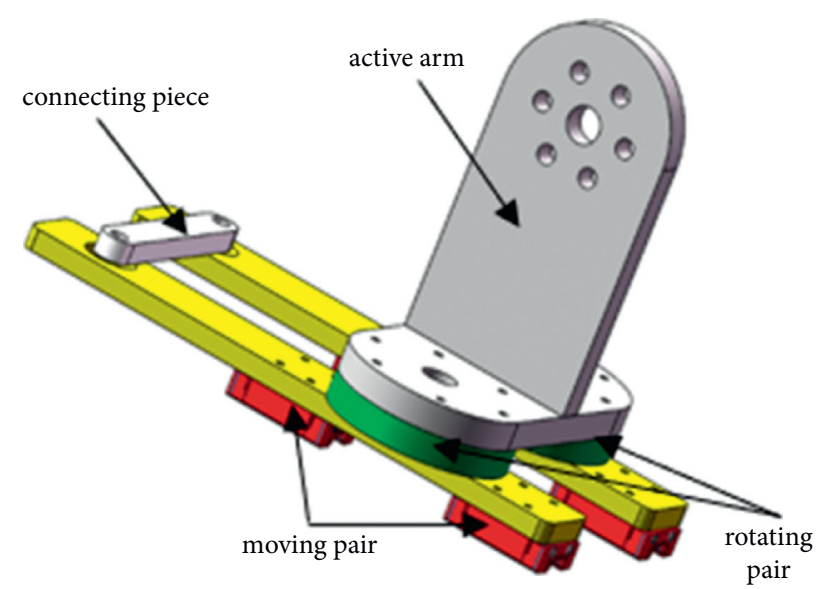

(b)

FIGURE 2: Composite branch structure diagram of (a) compound branch diagram and of (b) partial view.

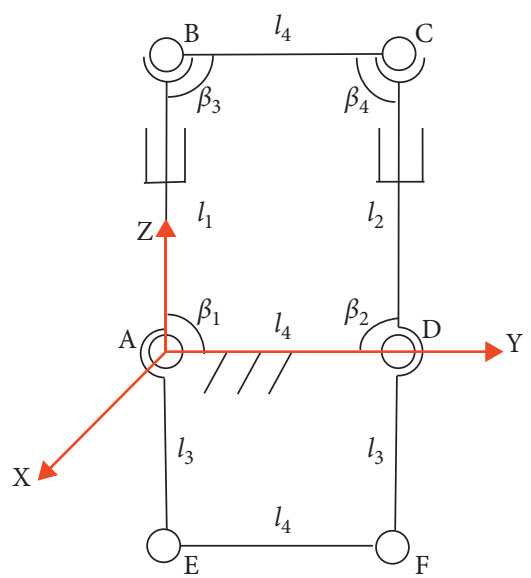

FIGURE 3: Composite branched chain mechanism diagram.

For the kinematic spiral system above, the inverse spiral can be obtained:

$$
\begin{aligned}
& \$_{1}^{r}=\left[\begin{array}{llllll}
1 & 0 & 0 & 0 & f & -e
\end{array}\right], \\
& \$_{2}^{r}=\left[\begin{array}{llllll}
0 & e & f & 0 & 0 & 0
\end{array}\right]^{T} .
\end{aligned}
$$

The two antihelices represent two constraints that limit movement parallel to the axis $X$ and the rod $\mathrm{AB}$ direction, respectively.

Because the two branches around have the same structure and configuration, as a result, they have the same antihelix and have the same two binding forces. For the $\mathrm{BC}$ rod of the moving platform, it bears a total of four binding forces, analyzing the correlation of its spiral, since the four binding forces are all line vectors, and the two line vectors of coplanar parallel are linearly independent. The two line vectors intersecting the plane are also linearly independent. This indicates that there are four independent constraints in the mechanism, which, respectively, constrain rotation about axis $X$ and axis $Z$, and movement along axis $X$ and rod $A B$ direction. In this way, the moving

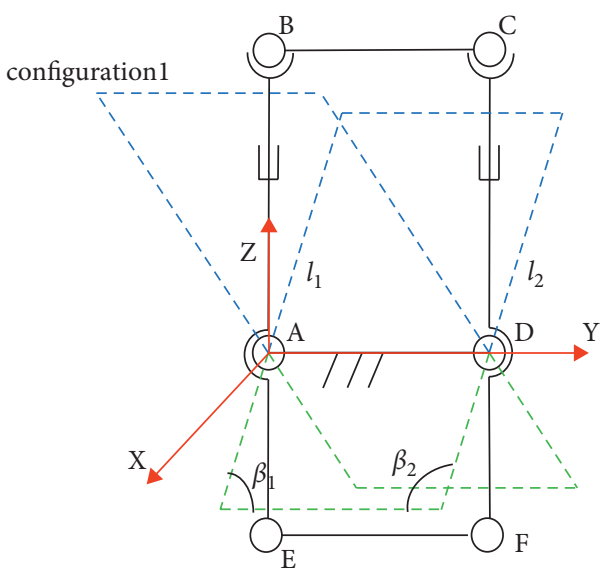

FIgURE 4: Configuration 1, parallelogram configuration.

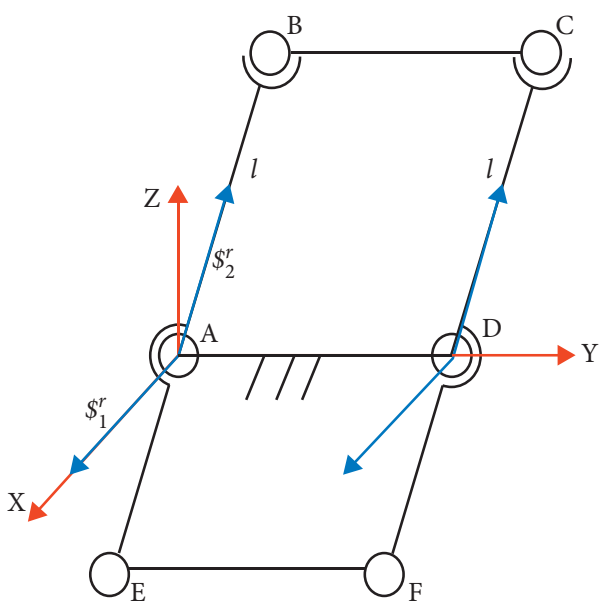

Figure 5: Parallelogram closed-loop subchain mechanism diagram.

platform has only the freedom of movement in the direction perpendicular to the $\operatorname{rod} A B$ and the freedom of rotation around the bar $\mathrm{BC}$. 
To sum up, as shown in Figure 4, configuration 1 is a synchronous telescopic parallelogram mechanism, so when $l$ is a variable, only one translational $\mathrm{DOF}$ along the $\mathrm{AB}$ direction is added for the moving platform. Therefore, in configuration 1, the DOF of the moving platform $\mathrm{BC}$ can be expressed by the basic motion screw system as follows:

$$
\begin{aligned}
& \$_{1}=\left[\begin{array}{llllll}
0 & 1 & 0 & 0 & 0 & 0
\end{array}\right]^{T}, \\
& \$_{2}=\left[\begin{array}{llllll}
0 & 0 & 0 & 0 & 1 & 0
\end{array}\right]^{T}, \\
& \$_{3}=\left[\begin{array}{llllll}
0 & 0 & 0 & 0 & 0 & 1
\end{array}\right]^{T} .
\end{aligned}
$$

As shown in Figure 6, when the mechanism is in configuration 2, there always have ${ }^{1} B^{1} C=A D,{ }^{1} B A / /{ }^{1} C D$. This moving platform only has one translational DOF in the ${ }^{1} B A$ direction, one in the direction parallel to the axis $X$, but rotational DOFs at the origin, and a local rotational DOF around the ${ }^{1} B^{1} C$ direction. The motion spiral system $\$_{s p}$ can be expressed as (6).

When the three branch chains are in configuration 1 of Figure 4, the DOF of the moving platform of each branch can be expressed by the moving screw system:

$$
\begin{aligned}
& \$_{1}=\left[\begin{array}{llllll}
0 & 1 & 0 & 0 & 0 & 0
\end{array}\right]^{T}, \\
& \$_{2}=\left[\begin{array}{llllll}
0 & 0 & 0 & 0 & 1 & 0
\end{array}\right]^{T}, \\
& \$_{3}=\left[\begin{array}{llllll}
0 & 0 & 0 & 0 & 0 & 1
\end{array}\right]^{T} .
\end{aligned}
$$

Therefore, the dynamic platform $\mathrm{BC}$ of the branch closedloop subchain can be expressed as one rotational and two translational DOFs. Thus, the 2RPS parallelogram mechanism can be regarded as a generalized pair ([PPR]) with 3 DOFs.

As shown in Figure 7, after replacing each closed-loop subchain with a generalized motion pair, it can be regarded as composed of three connecting rods and four motion pairs ( $R$ [PPR]), among which the first $R$ pair is connected with the frame in the mechanism, and the $R$ pair in the generalized motion pair is connected with the moving platform. When the branch coordinate system is taken on the equivalent mechanism of the mechanism, the axis $X$ can be along the normal surface of the closed-loop of the mechanism, and the axis $Y$ can be along the axis of the rotation pair on the fixator of the mechanism.

$$
\left\{\begin{array}{l}
\$_{s p 1}=\left[\begin{array}{llllll}
0 & 0 & 0 & 0 & \cos \beta_{1} & \sin \beta_{1}
\end{array}\right]^{T}, \\
\$_{s p 2}=\left[\begin{array}{lllll}
1 & 0 & 0 & 0 & \frac{l_{1} \sin \beta_{1}}{2}-\frac{l_{1} \cos \beta_{1}}{2}
\end{array}\right]^{T}, \\
\$_{s p 3}=\left[\begin{array}{llllll}
0 & l_{4}+\left(l_{2}-l_{1}\right) \cos \beta_{1} & \left(l_{2}-l_{1}\right) \sin \beta_{1}-l_{1} l_{4} \sin \beta_{1} & 0 & 0
\end{array}\right]^{T} .
\end{array}\right.
$$

The branch motion spiral system of this mechanism is

$$
\begin{aligned}
& \$_{i 1}=\left[\begin{array}{llllll}
0 & 1 & 0 & 0 & 0 & 0
\end{array}\right]^{T}, \\
& \$_{i 2}=\left[\begin{array}{llllll}
0 & 1 & 0 & d_{i 2} & 0 & f_{i 2}
\end{array}\right]^{T}, \\
& \$_{i 3}=\left[\begin{array}{llllll}
0 & 0 & 0 & 0 & 1 & 0
\end{array}\right]^{T}, \\
& \$_{i 4}=\left[\begin{array}{llllll}
0 & 0 & 0 & 0 & 0 & 1
\end{array}\right]^{T} .
\end{aligned}
$$

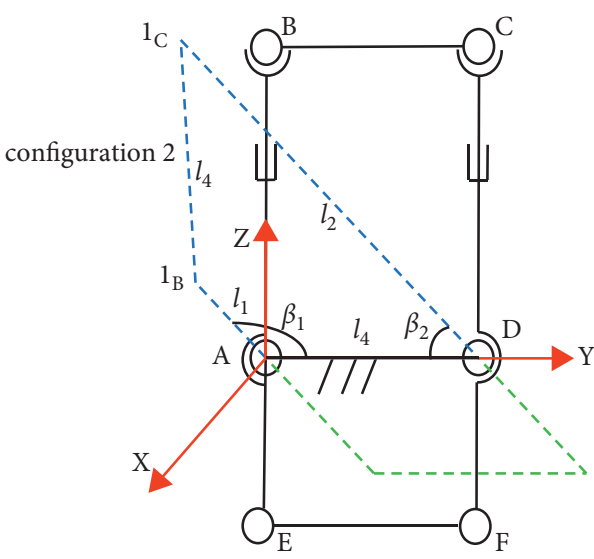

Figure 6: Configuration 2, isosceles trapezoid.

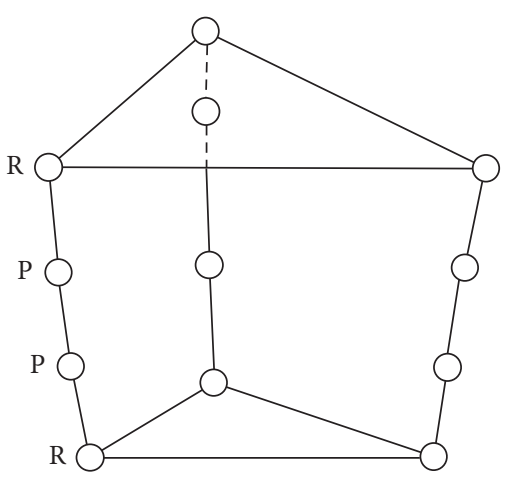

Figure 7: Mechanism equivalent diagram.

Its branch constraint antihelix system is

$$
\begin{aligned}
& \$_{i 1}^{r}=\left[\begin{array}{llllll}
0 & 0 & 0 & 1 & 0 & 0
\end{array}\right]^{T}, \\
& \$_{i 2}^{r}=\left[\begin{array}{llllll}
0 & 0 & 0 & 0 & 0 & 1
\end{array}\right]^{T} .
\end{aligned}
$$

Because these two antihelices are mutually perpendicular constraint pairs, their directions are perpendicular to the direction of the corresponding rotation axis on the fixator of the mechanism. Therefore, the moving platform of the mechanism bears six constraint pairs from three branches. Three of them are perpendicular to the fixed platform, forming a common constraint, namely, $\lambda=1$; the other three pairs are parallel to the fixed platform and are not parallel to each other, but they are linearly related to each other, and the maximum linearly independent number is 2 , so there is a redundant constraint, namely, $v=1$.

Thus, the final freedom of the agency is

$\mathrm{M}=d(n-g-1)+\sum_{i=1}^{g} f_{i}+v-\delta=5(11-12-1)+12+1=3$.

Considering that the moving platform of the mechanism is subjected to six constraint pairs from three branches, and their maximum linearly independent number is 3 , the three rotational DOFs of the moving platform are constrained. Therefore, when the branches are in singular configuration 
and configuration 1 , the mechanism is a $3 \mathrm{~T}$ parallel mechanism.

When the three branched chains are located in the singular configuration in Figure 3, each branched chain relative to configuration 1 has added an instantaneous rotational DOF around the axis $X$, three branched chains, and a total of three instantaneous rotational DOFs of moving platform because these three degrees of freedom are in different directions and space concurrent, thus linearly independent and mutual constraints.

Therefore, it can be known from the above analysis that, under the mutual constraints of the three branched chains, the moving platform of the mechanism will not rotate and not be in configuration 2, and each branch always keeps configuration 1. Therefore, rod $A B$ and rod $C D$ are synchronous telescopic components, and the whole mechanism is a $3 \mathrm{~T}$ parallel mechanism.

\section{Location Analysis}

4.1. Coordinate System Establishment. Each slave arm of the parallel mechanism is a parallelogram, and the distance between the two sliding rods is unchanged, so the structure can be simplified, as shown in Figure 8. The three points $L_{1}$, $L_{2}$, and $L_{3}$ are the vertices of the equilateral triangle, and the radius of its circumscribed circle is $\mathrm{R}$. With the center of the circle as the origin, the direction from which the center of the circle points to $L_{1}$ as the axis $X$, and the normal direction of the plane where the equilateral triangle is located as the axis $Z$, we establish the basic coordinate system O-XYZ according to the right-hand rule. The three points $\mathrm{N}_{1} \mathrm{~N}_{2} \mathrm{~N}_{3}$ are the vertices of an equilateral triangle whose circumscribed circle radius is $r$. Taking the center of the circle as the origin, and the three coordinate axes at the initial position are parallel to the basic coordinate system, we establish a dynamic coordinate system $\mathrm{O}^{\prime}-\mathrm{X}^{\prime} \mathrm{Y}^{\prime} \mathrm{Z}^{\prime}$. The length of the active $\operatorname{arm} L_{i} M_{i}$ is $l_{b}$, and the length of the slave arm $\mathrm{M}_{\mathrm{i}} \mathrm{N}_{\mathrm{i}}$ is $l_{a i}$, where $l_{a i}$ is a variable. The angle between the static platform and the active arm is $\theta_{i}$, and $\theta_{i}$ is the input parameter, where $i=1,2,3$. The coordinate of the center $\mathrm{O}^{\prime}$ of the moving platform is $\mathrm{O}^{\prime}(x, y, z)$, where $x, y$, and $z$ are output parameters.

4.2. Position Inverse Solution. According to the geometric relationship that always exists in the simplified mechanism: $\mathrm{LiMi} \perp \mathrm{MiNi}$, from the Pythagorean theorem

$$
\overline{L N}^{2}=\overline{L M}^{2}+\overline{M N}^{2},
$$

where the coordinate of point $L_{i}: L_{i}=R\left[\begin{array}{c}\cos \alpha_{i} \\ \sin \alpha_{i} \\ 0\end{array}\right]$; the coordinate of point $N_{i}: N_{i}=\left[\begin{array}{c}x+r \cos \alpha_{i} \\ y+r \sin \alpha_{i} \\ z\end{array}\right]$; the coordinate of point $M_{i}: \quad M_{i}=\left[\begin{array}{c}\left(l_{b} \cos \theta_{i}+R\right) \cos \alpha_{i} \\ \left(l_{b} \cos \theta_{i}+R\right) \sin \alpha_{i} \\ l_{b} \sin \theta_{i}\end{array}\right] ;$ and the rotation angle of the active arm is $\theta_{i}$, where $i=1,2,3$.

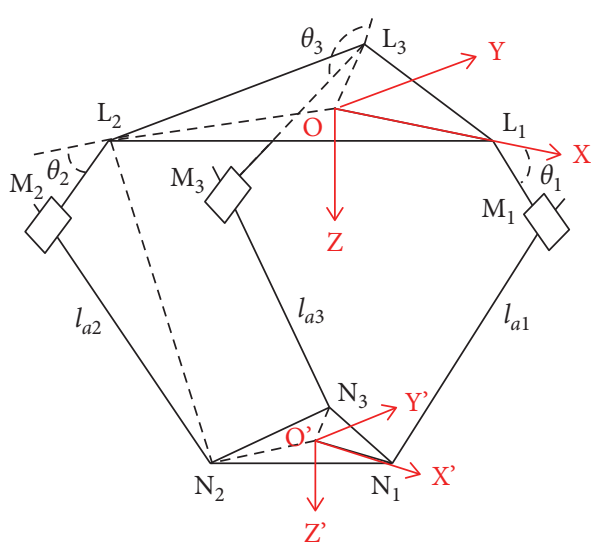

Figure 8: Coordinate system establishment.

Known from the coordinates,

$$
\begin{aligned}
\overline{L N}^{2}= & {\left[\left(x+r \cos \alpha_{i}\right)-R \cos \alpha_{i}\right]^{2} } \\
& +\left[\left(x+r \sin \alpha_{i}\right)-R \sin \alpha_{i}\right]^{2}+z^{2}, \\
\overline{L M}^{2}= & l_{b}^{2}, \\
\overline{M N}^{2}= & {\left[\left(x+r \cos \alpha_{i}\right)-\left(l_{b} \cos \theta_{i}+R\right) \cos \alpha_{i}\right]^{2} } \\
& +\left[\left(x+r \sin \alpha_{i}\right)-\left(l_{b} \cos \theta_{i}+R\right) \sin \alpha_{i}\right]^{2} \\
& +\left(z-l_{b} \sin \theta_{i}\right)^{2} .
\end{aligned}
$$

Substituting $\overline{L N}^{2}, \overline{L M}^{2}$, and $\overline{M N}^{2}$ into (9), after simplification, the equation of the rotation angle $\theta_{i}$ of the active arm and the center coordinates $\mathrm{O}^{\prime}(x, y, z)$ of the moving platform can be obtained as

$$
\left[x \cos \alpha_{i}+y \sin \alpha_{i}+(r-R)\right] \cos \theta_{i}+z \sin \theta_{i}-l_{b}=0 .
$$

The above equation can be abbreviated as

$$
Q_{i} \cos \theta_{i}+z \sin \theta_{i}-l_{b}=0,
$$

where

$$
Q_{i}=\left[x \cos \alpha_{i}+y \sin \alpha_{i}+(r-R)\right] .
$$

Let $t_{i}=\tan \left(\theta_{i} / 2\right)$, according to the universal equation, substituting (12) and sorting it out yield

$$
\left(Q_{i}+l_{b}\right) t_{i}^{2}-2 z t_{i}+\left(l_{b}-Q_{i}\right)=0 .
$$

The above equation can be solved by the one-dimensional quadratic equation:

$$
t_{i}=\frac{z \pm \sqrt{Q_{i}^{2}+z^{2}-l_{b}^{2}}}{Q_{i}+l_{b}}
$$

Get the inverse solution of the mechanism

$$
\theta_{i}=2 \arctan \left(t_{i}\right)
$$

4.3. Position Positive Solution. On the basis of the position inverse solution, according to (11), the simplified solution is 
$\cos \alpha_{i} \cos \theta_{i} x+\sin \alpha_{i} \cos \theta_{i} y+\sin \theta_{i} z+\cos \theta_{i}(r-R)-l_{b}=0$,

where

$$
i=1,2,3 \text {. }
$$

The above formula is simplified to

$$
A_{i} x+B_{i} y+C_{i} z+D_{i}=0(i=1,2,3)
$$

where

$$
\begin{aligned}
A_{i} & =\cos \alpha_{i} \cos \theta_{i}, \\
B_{i} & =\sin \alpha_{i} \cos \theta_{i}, \\
C_{i} & =\sin \theta_{i}, \\
D_{i} & =\cos \theta_{i}(r-R)-l_{b} .
\end{aligned}
$$

Equation (19) is eliminated and sorted out, and its positive solution is

$$
\left\{\begin{array}{l}
x=-\left(\mathrm{B}_{1} \mathrm{C}_{2} \mathrm{D}_{3}-\mathrm{B}_{1} \mathrm{C}_{3} \mathrm{D}_{2}-\mathrm{B}_{2} \mathrm{C}_{1} \mathrm{D}_{3}+\mathrm{B}_{2} \mathrm{C}_{3} \mathrm{D}_{1}+\mathrm{B}_{3} \mathrm{C}_{1} \mathrm{D}_{2}-\mathrm{B}_{3} \mathrm{C}_{2} \mathrm{D}_{1}\right) /\left(\mathrm{A}_{1} \mathrm{~B}_{2} \mathrm{C}_{3}-\mathrm{A}_{1} \mathrm{~B}_{3} \mathrm{C}_{2}-\mathrm{A}_{2} \mathrm{~B}_{1} \mathrm{C}_{3}+\mathrm{A}_{2} \mathrm{~B}_{3} \mathrm{C}_{1}+\mathrm{A}_{3} \mathrm{~B}_{1} \mathrm{C}_{2}-\mathrm{A}_{3} \mathrm{~B}_{2} \mathrm{C}_{1}\right) \\
y=-\left(\mathrm{A}_{1} \mathrm{C}_{2} \mathrm{D}_{3}-\mathrm{A}_{1} \mathrm{C}_{3} \mathrm{D}_{2}-\mathrm{A}_{2} \mathrm{C}_{1} \mathrm{D}_{3}+\mathrm{A}_{2} \mathrm{C}_{3} \mathrm{D}_{1}+\mathrm{A}_{3} \mathrm{C}_{1} \mathrm{D}_{2}-\mathrm{A}_{3} \mathrm{C}_{2} \mathrm{D}_{1}\right) /\left(\mathrm{A}_{1} \mathrm{~B}_{2} \mathrm{C}_{3}-\mathrm{A}_{1} \mathrm{~B}_{3} \mathrm{C}_{2}-\mathrm{A}_{2} \mathrm{~B}_{1} \mathrm{C}_{3}+\mathrm{A}_{2} \mathrm{~B}_{3} \mathrm{C}_{1}+\mathrm{A}_{3} \mathrm{~B}_{1} \mathrm{C}_{2}-\mathrm{A}_{3} \mathrm{~B}_{2} \mathrm{C}_{1}\right) \\
Z=-\left(\mathrm{A}_{1} \mathrm{~B}_{2} \mathrm{D}_{3}-\mathrm{A}_{1} \mathrm{~B}_{3} \mathrm{D}_{2}-\mathrm{A}_{2} \mathrm{~B}_{1} \mathrm{D}_{3}+\mathrm{A}_{2} \mathrm{~B}_{3} \mathrm{D}_{1}+\mathrm{A}_{3} \mathrm{~B}_{1} \mathrm{D}_{2}-\mathrm{A}_{3} \mathrm{~B}_{2} \mathrm{D}_{1}\right) /\left(\mathrm{A}_{1} \mathrm{~B}_{2} \mathrm{C}_{3}-\mathrm{A}_{1} \mathrm{~B}_{3} \mathrm{C}_{2}-\mathrm{A}_{2} \mathrm{~B}_{1} \mathrm{C}_{3}+\mathrm{A}_{2} \mathrm{~B}_{3} \mathrm{C}_{1}+\mathrm{A}_{3} \mathrm{~B}_{1} \mathrm{C}_{2}-\mathrm{A}_{3} \mathrm{~B}_{2} \mathrm{C}_{1}\right) .
\end{array}\right.
$$

\section{Velocity Analysis and Jacobian Matrix}

The Jacobian matrix, also known as the first-order motion influence coefficient, is the mapping between the operating speed of the machine and the joint speed and can also be regarded as the transmission ratio of the motion speed from the joint space to the operating space. The end speed of the parallel mechanism and the input angular speed are expressed as

$$
\dot{X}=J \dot{\theta},
$$

where $\dot{X}=\left[\begin{array}{lll}\dot{x} & \dot{y} & \dot{z}\end{array}\right]^{T}$ represents the end velocity vector , $\dot{\theta}=\left[\begin{array}{lll}\dot{\theta}_{1} & \dot{\theta}_{2} & \dot{\theta}_{3}\end{array}\right]^{T}$ represents the driving speed vector, and $J$ represents the Jacobian matrix of the parallel mechanism.

By equation (11), let

$$
F_{i}=\left[x \cos \alpha_{i}+y \sin \alpha_{i}+(r-R)\right] \cos \theta_{i}+z \sin \theta_{i}-l_{b}=0 .
$$

Solve the derivative of the above equation with respect to time

$$
\frac{\partial F_{i}}{\partial x} \dot{x}+\frac{\partial F_{i}}{\partial y} \dot{y}+\frac{\partial F_{i}}{\partial z} \dot{z}+\frac{\partial F_{i}}{\partial \theta_{i}} \dot{\theta}=0 .
$$

Sorted it out

$$
A \dot{X}=B \dot{\theta} .
$$

It can be concluded that the Jacobian matrix of the parallel mechanism is

$$
J=A^{-1} B
$$

Here, in (24)

$$
\begin{aligned}
& \frac{\partial F_{i}}{\partial x}=\cos \alpha_{i} \cos \theta_{i}, \\
& \frac{\partial F_{i}}{\partial y}=\sin \alpha_{i} \cos \theta_{i}, \\
& \frac{\partial F_{i}}{\partial z}=\sin \theta_{i}, \\
& \frac{\partial F_{i}}{\partial \theta_{i}}=z \cos \theta_{i}-\left(x \cos \alpha_{i}+y \sin \alpha_{i}+r-R\right) \sin \theta_{i} .
\end{aligned}
$$

Here, in (25)

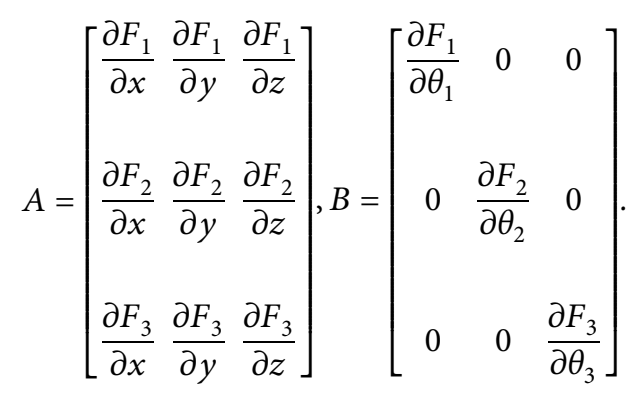

\section{Singular Configuration Analysis}

The singularity of the mechanism is related to the stability of the mechanism's performance. When the mechanism is in a singular position, there will be one or more degrees of freedom lost, causing the moving platform to be uncontrollable in certain directions. The methods to analyze the singular configuration of the mechanism mainly include kinematics, geometry, and algebra. Among them, the algebraic method to solve the singular configuration of the parallel mechanism is to find whether the Jacobian matrix is full rank. When $J=A^{-1} B=0$, the parallel mechanism will show singular configuration.

According to the Jacobian matrix of the parallel mechanism, the singular configuration of the mechanism in this paper is analyzed, and (26) is solved. When the angle between the static platform and the active arm is $\pi / 2$, we can get $J=0$. The mechanism has a singular configuration. As shown in Figure 9, at this time, the branch chain constraint of the parallel mechanism increases, and the degree of freedom of the moving platform decreases.

\section{Workspace Analysis}

The workspace of the parallel mechanism refers to the collection of all points that the reference point of the endeffector can reach in the workspace. This paper mainly adopts the Monte Carlo method that a large number of random numbers are used to determine enough random joints. The reference points of all end actuators of the 


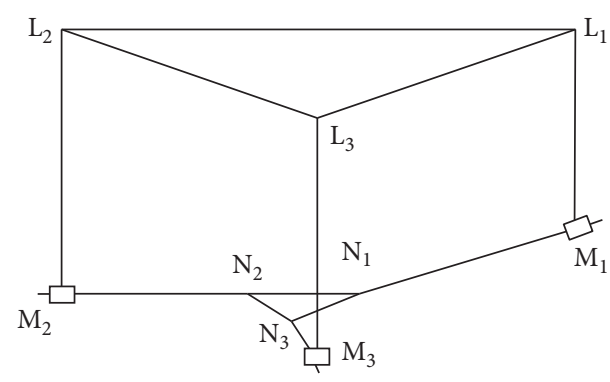

FIGURE 9: Singularity diagram.

mechanism are drawn to form the workspace of the mechanism.

The position equation is combined with the known parameters of the parallel mechanism: the radius of the circumscribed circle of the static platform is $R=120 \mathrm{~mm}$, the radius of the circumscribed circle of the movable platform is $r=34.64 \mathrm{~mm}$, and the length of the active arm member is $l_{b}=99.75 \mathrm{~mm}$, and the angle $\theta_{i}$ between the static platform and the active arm is defined as $\left[20^{\circ}, 75^{\circ}\right]$. The maximum length of the variable-length links is $350 \mathrm{~mm}$. In this range, by setting a large number of random variables to substitute the position forward solution, through MATLAB programming, the workspace diagram of the parallel mechanism is obtained, as shown in Figure 10. Figures 10(a)-10(c) show three views of different workspaces, and Figure 10(d) shows a three-dimensional view of the workspace.

\section{Trajectory Planning}

Trajectory planning can be summarized as follows: according to the needs of the robot in actual working conditions, plan a trajectory or path that can complete the work task within the reach of its end-effector. Reasonable trajectory planning can not only shorten the motion cycle and improve work efficiency but also reduce structural vibration and extend the service life.

8.1. Joint Space Trajectory Planning. To plan the trajectory of the parallel mechanism, in order to ensure the smooth movement of each joint and prevent sudden changes in acceleration, this article uses a fifth-degree polynomial for interpolation

$$
\left\{\begin{array}{l}
s(t)=a_{0}+a_{1} t+a_{2} t^{2}+a_{3} t^{3}+a_{4} t^{4}+a_{5} t^{5} \\
v(t)=a_{1}+2 a_{2} t+3 a_{3} t^{2}+4 a_{4} t^{3}+5 a_{5} t^{4} \\
a(t)=2 a_{2}+6 a_{3} t+12 a_{4} t^{2}+20 a_{5} t^{3}
\end{array}\right.
$$

where $s, v$, and $a$ are the path length, velocity, and acceleration, respectively. $a_{0}, a_{1}, \ldots, a_{5}$ are the undetermined coefficients of the polynomial, and $t$ is the time.

In order to ensure the smooth operation of the parallel mechanism, the following boundary constraints are specified:

$$
\begin{gathered}
s(0)=0, v(0)=0, a(0)=0, \\
s\left(t_{f}\right)=s_{f}, v\left(t_{f}\right)=0, a\left(t_{f}\right)=0,
\end{gathered}
$$

where $t_{f}$ represents the terminal time of the trajectory and $s_{f}$ is the total length of the planned path of the trajectory.

Substituting the above boundary conditions, the undetermined coefficients can be solved, and the trajectory planning equation is obtained as follows:

$$
\left\{\begin{array}{l}
s(t)=\frac{10 s_{f}}{t_{f}^{3}} t^{3}-\frac{15 s_{f}}{t_{f}^{4}} t^{4}+\frac{6 s_{f}}{t_{f}^{5}} t^{5}, \\
v(t)=\frac{30 s_{f}}{t_{f}^{3}} t^{2}-\frac{60 s_{f}}{t_{f}^{4}} t^{3}+\frac{30 s_{f}}{t_{f}^{5}} t^{4}, \\
a(t)=\frac{60 s_{f}}{t_{f}^{3}} t-\frac{180 s_{f}}{t_{f}^{4}} t^{2}+\frac{30 s_{f}}{t_{f}^{5}} t^{3}
\end{array}\right.
$$

8.2. Coordinate Space Trajectory Planning. The trajectory planning of the parallel mechanism needs to involve the relationship of the path and the time of its motion. This paper mainly introduces a kind of door-shaped trajectory, which can meet the needs of picking and placing operations in the industry.

As shown in Figure 11, $\mathrm{P}_{0}$ is the picking point and $\mathrm{P}_{5}$ is the placing point. The trajectory is composed of three straight lines and two arcs. The two transition arcs can effectively avoid sudden changes in speed and acceleration.

Assuming that the door-shaped trajectory is in the $\mathrm{XOZ}$ plane, the initial position $\mathrm{P}_{0}\left(x_{0}, 0, z_{0}\right)$ is given. Interpolate the straight line and circular arc in Figure 11, respectively, to obtain the door-shaped trajectory motion (32).

$\varphi_{1}=\left(s\left(t_{i}\right)-h / r\right)$ and $\varphi_{2}=\left(s\left(t_{i}\right)-h-(1 / 2) \pi r_{p}-l / r_{p}\right)$. $t_{1} \ldots t_{5}$ correspond to the time of arrival at $P_{1} \ldots P_{5}$, respectively. $t_{i}$ is any time in the cycle.

\section{Simulation Analysis}

According to the analysis of trajectory planning, the trajectory of the end-effector of the parallel mechanism is divided into three straight sections and two circular arcs. The picking position is $\mathrm{P}_{0}(-45,0,240)$, and the placing position is $\mathrm{P}_{5}(45,0,240) . h=30 \mathrm{~mm}, l=70 \mathrm{~mm}, \quad r_{p}=10 \mathrm{~mm}$, and $t_{f}=3 s$.

In the initial position, the default initial angle $\theta_{i}$, in SolidWorks, between the static platform and the active arm is 0. Sampling points in these five trajectories, and then performing motion analysis in SolidWorks, let the end-effector move along the door-shaped trajectory, and draw the curve of the angle $\theta_{i}$ on the three composite branched chains with time $t$, as shown in Figure 12. 


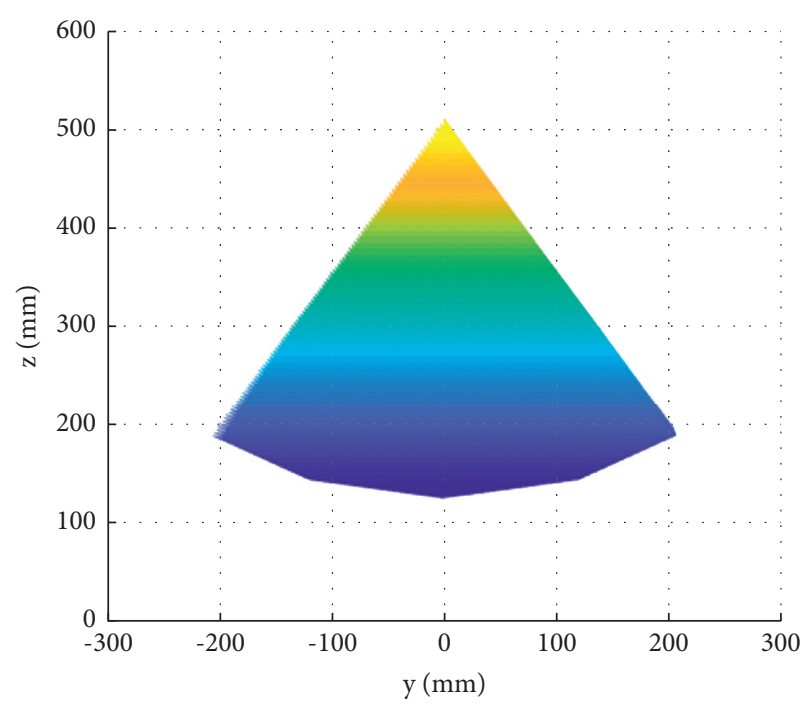

(a)

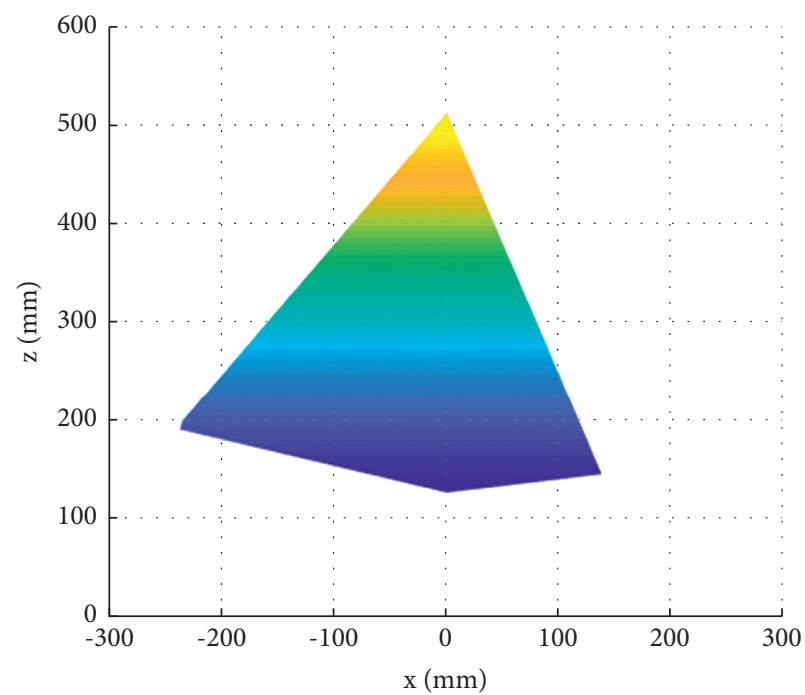

(c)

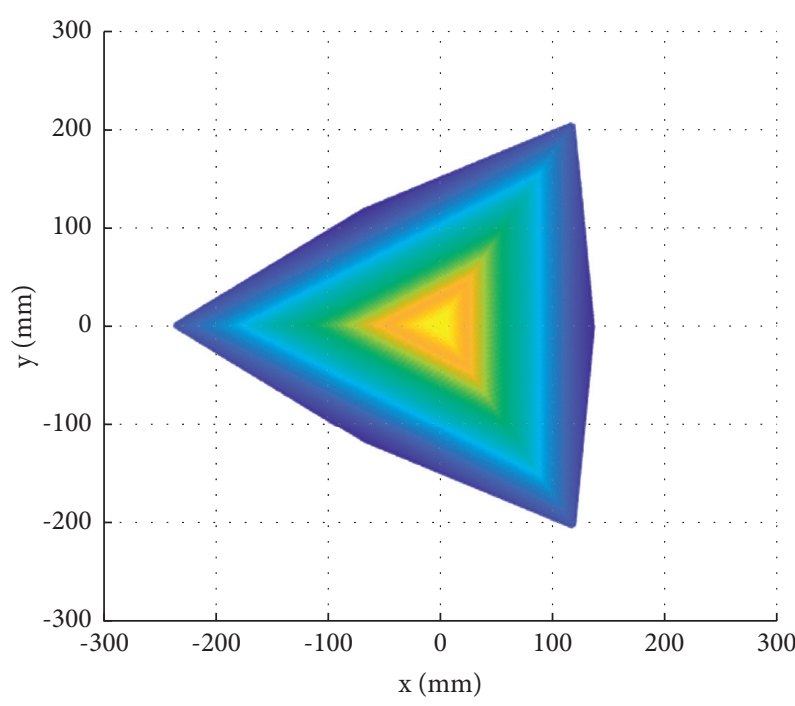

(b)

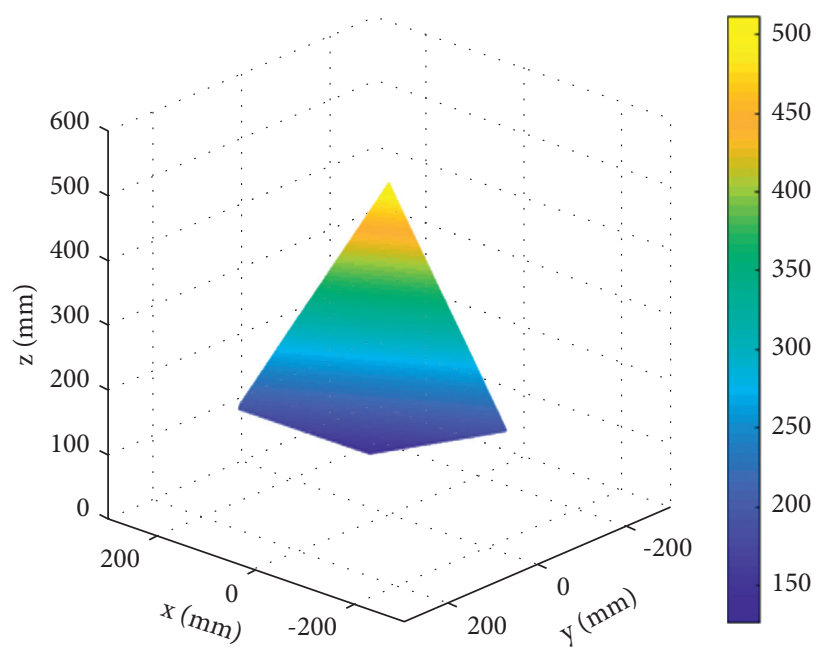

(d)

Figure 10: Schematic diagram of workspace. (a) y-o-z view, (b) x-o-y view, (c) x-o-z view, and (d) workspace diagram.

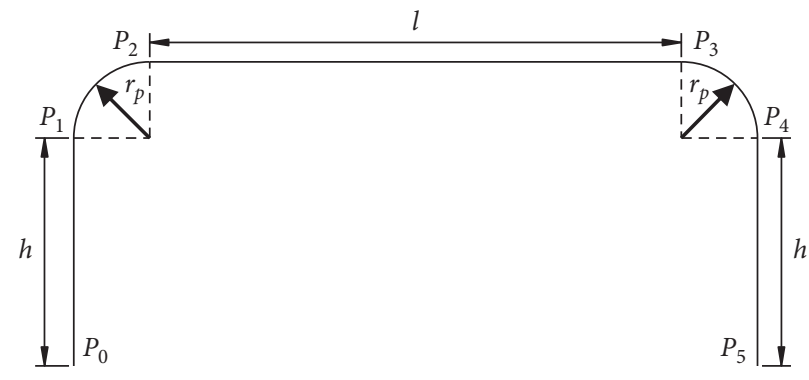

FIGURE 11: Door-shaped trajectory diagram. 


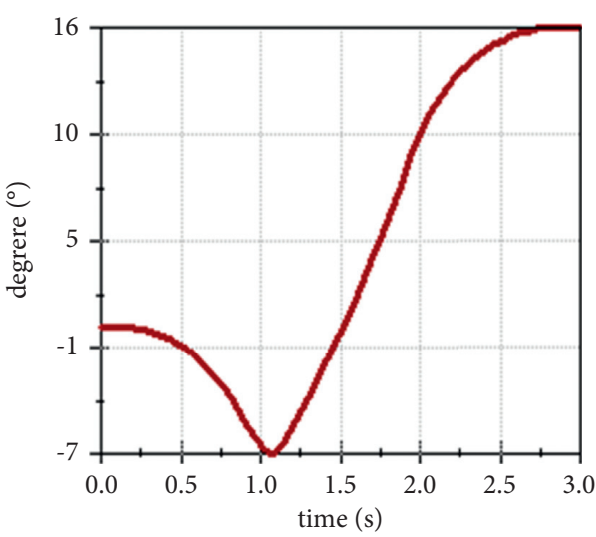

(a)

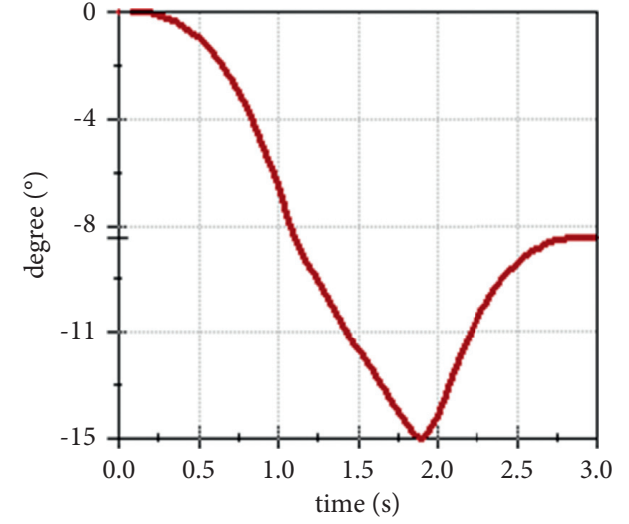

(b)

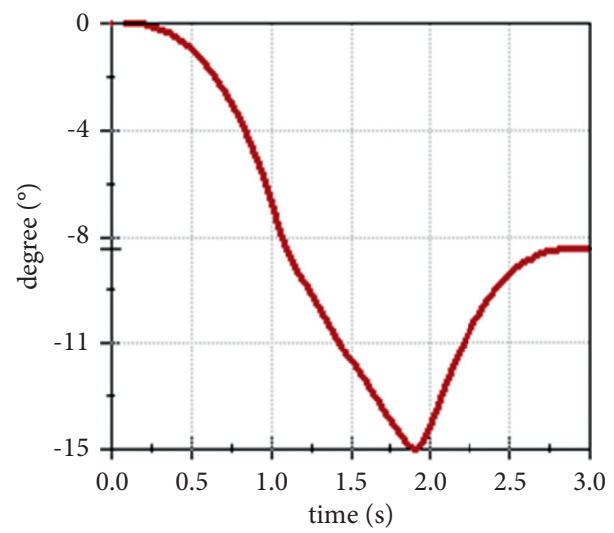

(c)

FIgURE 12: Variation curve of different branch chain angles 1. (a) Branched chain 1, (b) branched chain 2, and (c) branched chain 3.

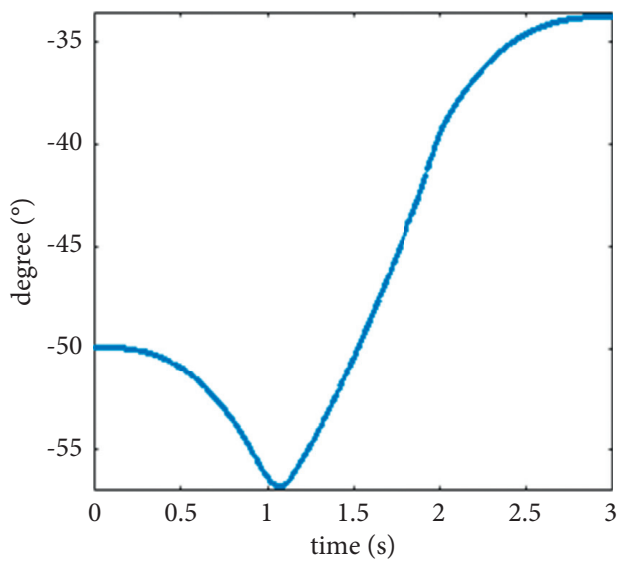

(a)

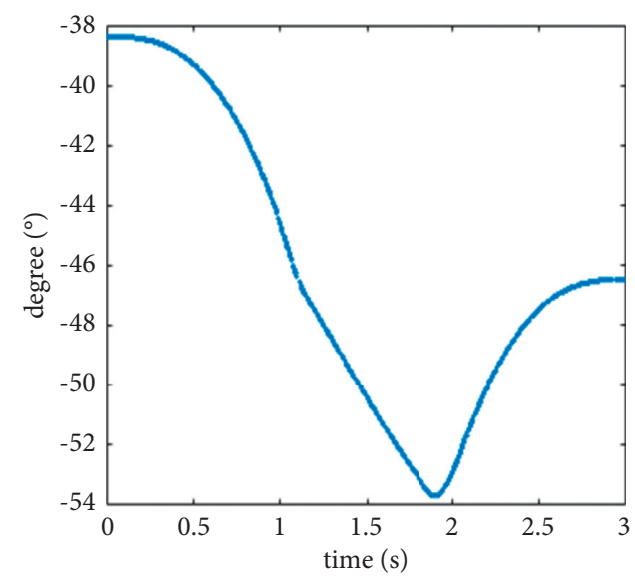

(b)

Figure 13: Continued. 


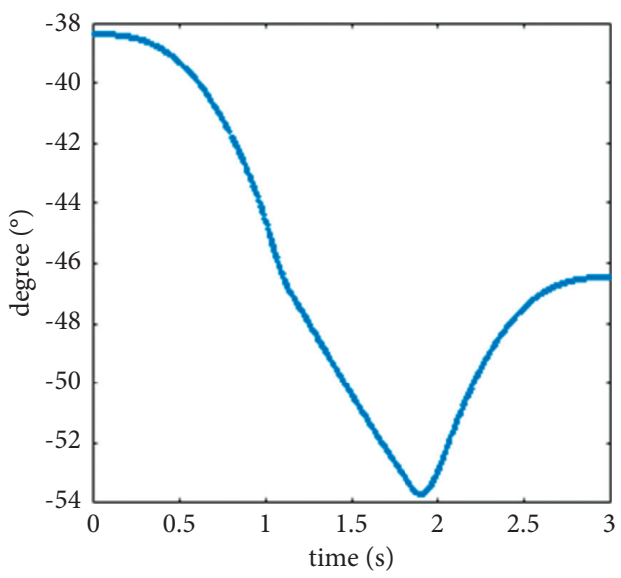

(c)

FiguRE 13: Variation curve of different branch chain angles 2. (a) Branched chain 1, (b) branched chain 2, and (c) branched chain 3.

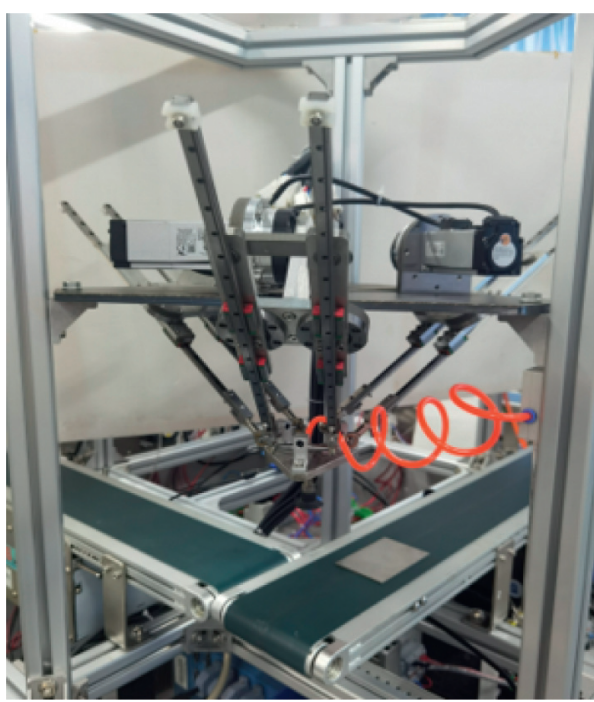

(a)
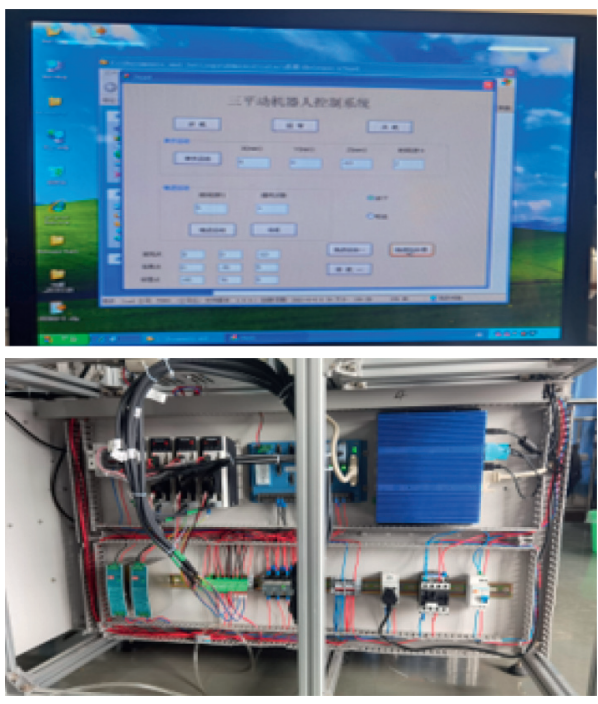

(b)

FIgURE 14: 3T parallel mechanism. (a) Overall picture of the mechanism and (b) operation interface and control system.

$$
\begin{cases}(x, y, z)=\left(x_{0}, 0, z_{0}-s\left(t_{i}\right)\right) & t_{0}<t_{i} \leq t_{1} \\ (x, y, z)=\left(x_{0}+r_{p}-r_{p} \cos \varphi_{1}, 0, z_{0}-h-r_{p} \sin \varphi_{1}\right) & \mathrm{t}_{1}<t_{i} \leq t_{2} \\ (x, y, z)=\left(x_{0}+r_{p}+s\left(t_{i}\right)-0.5 \pi r_{p}-h, 0, z_{0}-h-r_{p}\right) & t_{2}<t_{i} \leq t_{3} \\ (x, y, z)=\left(x_{0}+r_{p}+l+r_{p} \sin \varphi_{2}, 0, z_{0}-h-r_{p} \cos \varphi_{2}\right) & \mathrm{t}_{3}<t_{i} \leq t_{4} \\ (x, y, z)=\left(x_{0}+2 r_{p}+l, 0, z_{0}-s_{f}+s\left(t_{i}\right)\right) & t_{4}<t_{i} \leq t_{5}\end{cases}
$$

We simulate the gate trajectory in MATLAB, using the door-shaped trajectory equation in trajectory planning. Then, through the inverse solution of position, we program to obtain the change curve graph of the upper angle $\theta_{i}$ of the three composite branch chains with time $t$, as shown in Figure 13. By comparing with Figure 12, it can be seen that the angle change rates of the three branched chains are the same.

\section{Prototype and Experiments}

The $3 \mathrm{~T}$ parallel mechanism is composed of a mechanical body and a control system. The mechanical body is mainly composed of structural parts, motors, and suction cups, as shown in Figure 14(a). The control system is mainly composed of industrial computer, motion controller, servo driver, and servo part, as shown in Figure 14(b). The servo 


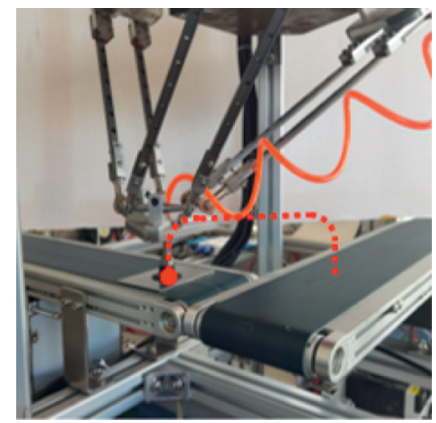

(a)

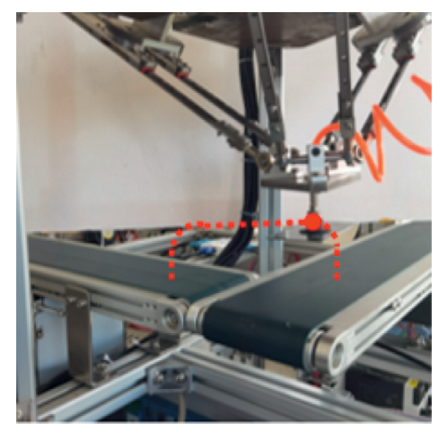

(d)

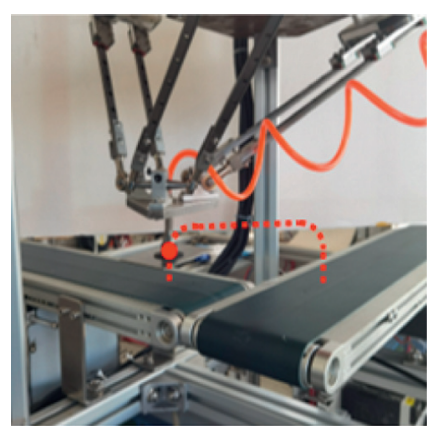

(b)

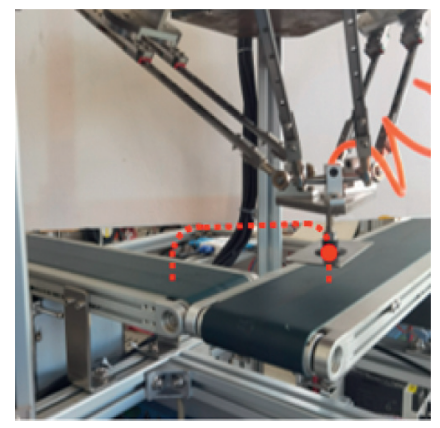

(e)

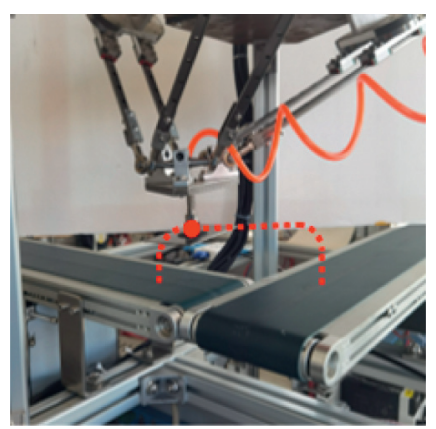

(c)

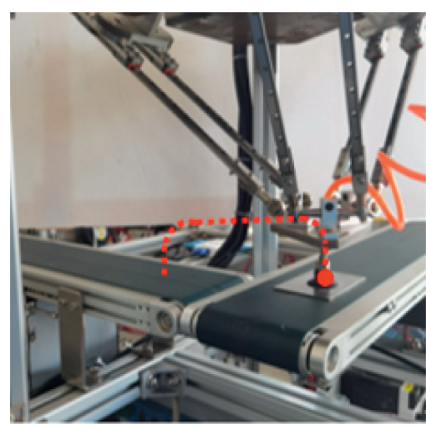

(f)

Figure 15: Prototype door trajectory movement diagram.

TABle 1: Parameters of trajectory point.

\begin{tabular}{lc}
\hline Tracing point & Coordinate $(\mathrm{mm})$ \\
\hline P0 & $(-45,0,240)$ \\
P1 & $(-45,0,210)$ \\
P2 & $(-35,0,200)$ \\
P3 & $(35,0,200)$ \\
P4 & $(45,0,210)$ \\
P5 & $(45,0,240)$ \\
\hline
\end{tabular}

systems of the three branch chains coordinate with each other to determine the movement of the moving platform of the parallel mechanism. The industrial computer communicates with the controller through the PCIe interface and sends control information to the controller. The controller outputs pulses to control the speed of the servo motor, thereby realizing the motion control of the moving platform.

Running the experiment according to the path in the trajectory planning, a sample is tested from $P 0 \cdots P 5$ according to the trajectory of door shape. The door-shaped trajectory motion diagram of the whole experiment is shown in Figure 15, and the red dots represent the endpoints of the effector of the parallel mechanism. The relevant parameters of all trajectory points in the basic coordinate system are shown in Table 1.

\section{Conclusion}

(1) A novel 3T parallel mechanism with variable-length parallelogram closed-loop subchain was designed. Based on the screw theory, the degree of freedom of the mechanism was analyzed, and the number and nature of the mechanism were $3 \mathrm{~T}$.
(2) The kinematics analysis of the mechanism was carried out, including the positive and inverse solution of position, speed, workspace, and singularity. The trajectory planning of the mechanism was completed, and the rationality of the mechanism design and the correctness of the theoretical analysis were verified through simulation analysis and prototype testing.

(3) Compared with other mechanisms with variablelength parallelogram closed-loop subchains, the mechanism described in this article has the advantages of simple structure and the drive can be installed on a static platform. Therefore, the mechanism has a good application prospect in light load and fast picking occasions.

\section{Data Availability}

All the data in this paper are obtained by calculation. The data used to support the findings of this study are available from the corresponding author upon request.

\section{Conflicts of Interest}

The authors declare that there are no potential conflicts of interest with respect to the research, authorship, and/or publication of this article.

\section{Acknowledgments}

This work was supported by the Key Scientific and Technological Support Topics in Jiangxi Province under Grant no. 20192BBE50084. 


\section{References}

[1] D. Andrioaia, G. Stan, M. Funaru, and C. Obreja, "The influence of kinematic joints clearances on the positioning accuracy of 3dof delta parallel robots," Applied Mechanics and Materials, vol. 371, pp. 406-410, 2013.

[2] J. P. Merlet, Parallel Robots, Springer, Berlin, Germany, 2006.

[3] R. Clavel, "Device for displacing and positioning an element in space," US. Patent 4976582, 1985.

[4] J. Lin, C. H. Luo, and K. H. Lin, "Design and implementation of a new delta parallel robot in robotics competitions," International Journal of Advanced Robotic Systems, vol. 12, no. 10, pp. 236-239, 2015.

[5] N. Liu and J. Wu, "Kinematics and application of a hybrid industrial robot-delta-RST," Sensors \& Transducers, vol. 169, no. 4 , p. 186, 2014

[6] J. Gallardo-Alvarado, A. L. Balmaceda-Santamaría, and E. Castillo-Castaneda, "An application of screw theory to the kinematic analysis of a Delta-type robot," Journal of $\mathrm{Me}$ chanical Science and Technology, vol. 28, no. 92, pp. 37853792, 2014.

[7] C. E. Boudjedir, D. Boukhetala, and M. Bouri, "Nonlinear PD plus sliding mode control with application to a parallel delta robot," Journal of Electrical Engineering, vol. 69, no. 5, pp. 329-336, 2018.

[8] J. Hervé, "Group mathematics and parallel link mechanisms," Proceedings of IMACS/SICE International Symposium on Robotics, Mechatronics and Manufacturing Systems, vol. 92, pp. 459-464, 1992.

[9] L. W. Tsai, Robot Analysis: The Mechanics of Serial and Parallel Manipulators, John Wiley \& Sons, New York, NY, USA, 1999.

[10] L. W. Tsai and R. Stamper, "A parallel manipulator with only translational degrees of freedom," in Proceedings of the International Design Engineering Technical Conferences and Computers and Information in Engineering Conference, vol. 97584, Article ID V02BT02A018, Irvine CA, US, August 1996.

[11] X.-J. Liu, Z.-L. Jin, and F. Gao, "Optimum design of 3-DOF spherical parallel manipulators with respect to the conditioning and stiffness indices," Mechanism and Machine Theory, vol. 35, no. 9, pp. 1257-1267, 2000.

[12] X. J. Liu, J. i. Jeong, and J. Kim, "A three translational DoFs parallel cube-manipulator,” Robotica, vol. 21, no. 6, pp. 645-653, 2003.

[13] X. J. Liu, "Optimal kinematic design of a three translational DoFs parallel manipulator," Robotica, vol. 24, no. 2, pp. 239-250, 2006.

[14] Q. Zou, D. Zhang, X. Luo, G. Huang, L. Li, and H. Zhang, "Enumeration and optimum design of a class of translational parallel mechanisms with prismatic and parallelogram joints," Mechanism and Machine Theory, vol. 150, Article ID 103846, 2020.

[15] Q. Zou, D. Zhang, S. Zhang, and X. Luo, "Kinematic and dynamic analysis of a 3-DOF parallel mechanism," International Journal of Mechanics and Materials in Design, vol. 17, pp. 1-13, May. 2021.

[16] G. Gogu, Structural Synthesis of Parallel Robots: Part 7 Limbs with Three Degrees of Connectivity, Springer, Berlin, Germany, 2008.

[17] Y. Yang, W. Zhang, H. Pu, and Y. Peng, "A class of symmetrical 3T, 3T-1R, and 3R mechanisms with parallel linear motion elements," Journal of Mechanisms and Robotics, vol. 10, no. 5, pp. 1-18, October. 2018.
[18] Y. Yang, Y. Peng, H. Pu et al., "Deployable parallel lowermobility manipulators with scissor-like elements," Mechanism and Machine Theory, vol. 135, pp. 226-250, 2019.

[19] Y. Yang, Y. Tang, H. Chen, Y. Peng, and H. Pu, "Mechanism design and parameter optimization of a new asymmetric translational parallel manipulator," Mechanical Sciences, vol. 10, no. 1, pp. 255-272, 2019.

[20] H. Wang, R. Yang, X. Kang, Z. Jiang, and X. Zou, "A novel family of parallel mechanisms with synchronous telescopic parallelograms," IEEE Access, vol. 7, pp. 184808-184824, 2019.

[21] R. Kelaiaia, O. Company, and A. Zaatri, "Multiobjective optimization of a linear Delta parallel robot," Mechanism and Machine Theory, vol. 50, pp. 159-178, 2012.

[22] A. Hernández, O. Altuzarra, O. Salgado, C. Pinto, and V. Petuya, "Designing parallel manipulators: from specifications to a real prototype," Industrial Robot: International Journal, vol. 39, no. 5, pp. 500-512, August. 2012. 